\title{
Malformação arteriovenosa incomum em artroplastia total do quadril: Relato de caso de um perioperatório de curso complicado
}

\section{Unusual Arteriovenous Malformation in Total Hip Arthroplasty: Case Report of a Complicated Perioperative Course}

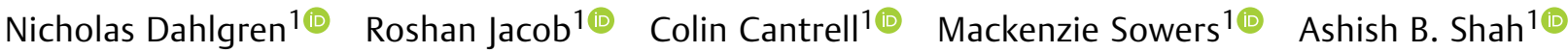 \\ Sameer Mahadeorao Naranje ${ }^{10}$ \\ 1 Universidade do Alabama em Birmingham, Birmingham, Alabama, \\ Endereço para correspondência Sameer Mahadeorao Naranje, MD, \\ University of Alabama at Birmingham, 1313 13th Street South, \\ Birmingham, AL, Estados Unidos (e-mail: snaranje@uabmc.edu). \\ Rev Bras Ortop
}

\begin{abstract}
Resumo
Palavras-chave

- malformações arteriovenosas

- artroplastia de quadril

- malformações vasculares

Não é comum encontrar malformações arteriovenosas (MAV) durante a artroplastia total do quadril (ATQ). Relatamos o presente caso para chamar a atenção para a possibilidade de uma MAV durante a abordagem anterior direta (AAD) para ATQ, que se não for considerada, pode levar a uma miríade de complicações relacionadas ao sangramento excessivo. Uma mulher de 81 anos foi apresentada ao pronto-socorro com fratura no pescoço do fêmur esquerdo. Ela optou por se submeter a uma artroplastia total do quadril (ATQ) através da AAD. Vasos sanguíneos aparentemente anormais estavam presentes perto dos ramos circunflexos ascendentes, proporcionando dificuldade em alcançar hemostasia. A perda excessiva de sangue foi notada e a paciente recebeu uma unidade de glóbulos vermelhos embalados durante a operação. Hemoglobina e hematócrito caíram nos dias seguintes à cirurgia, exigindo várias transfusões adicionais de produtos sanguíneos. Quando a paciente reclamou de inchaço progressivo na perna esquerda no terceiro dia pós-operatório, a tomografia computadorizada revelou hematomas grandes dentro dos adutores esquerdos e do músculo iliopsoas esquerdo. A extravasão ativa foi identificada a partir de um ramo da artéria femoral esquerda, bem como de uma fístula arteriovenosa (FAV) nesta área. $\mathrm{O}$ sangramento foi controlado por embolização seletiva da bobina endovascular. A partir do conhecimento atual, esta é a primeira descoberta intraoperatória relatada de MAC congênita com desenvolvimento subsequente de FAV pós-operatória e hematomas sintomáticos associados no cenário de ATQ utilizando a AAD. O reconhecimento precoce e a intervenção de malformações vasculares são essenciais para prevenir possíveis complicações cirúrgicas de membros ou de risco de vida.
\end{abstract}

recebido

08 de Julho de 2020

aceito

02 de Dezembro de 2020
DOI https://doi.org/

10.1055/s-0040-1722586. ISSN 0102-3616. (c) 2021. Sociedade Brasileira de Ortopedia e Traumatologia. All rights reserved.

This is an open access article published by Thieme under the terms of the Creative Commons Attribution-NonDerivative-NonCommercial-License, permitting copying and reproduction so long as the original work is given appropriate credit. Contents may not be used for commercial purposes, or adapted, remixed, transformed or built upon. (https://creativecommons.org/ licenses/by-nc-nd/4.0/)

Thieme Revinter Publicações Ltda., Rua do Matoso 170, Rio de Janeiro, RJ, CEP 20270-135, Brazil 


\begin{abstract}
It is not common to encounter arteriovenous malformations (AVMs) during total hip arthroplasty (THA). We report the present case to draw attention to the possibility of an AVM during the direct anterior approach (DAA) for THA, which, if not borne in mind, may lead to the myriad of complications related to excessive bleeding. An 81-year-old female presented to the emergency department with a left femoral neck fracture. She elected to undergo a THA via the DAA. Abnormal appearing blood vessels were present near the ascending circumflex branches, which provided difficulty in achieving hemostasis. Excessive blood loss was noted, and the patient received one unit of packed red blood cells during the operation. Hemoglobin and hematocrit dropped in the days following surgery, requiring several additional transfusions of blood products. When the patient complained of progressive left leg swelling on postoperative day 3 , a computed tomography revealed large hematomas within the left adductors and the left iliopsoas muscle. Active extravasation was identified arising from a branch of the left profunda femoral artery, as well as an arteriovenous fistula (AVF) in this area. Bleeding was controlled by selective endovascular coil embolization. As of current knowledge, this is the first reported intraoperative discovery of congenital arteriovenous malformation (AVM) with subsequent development of postoperative arteriovenous fistula and associated symptomatic hematomas in the setting of THA using the DAA. Early recognition and intervention of vascular malformations is essential in preventing potential limb- or life-threatening surgical complication.
\end{abstract}

\section{Introdução}

A artroplastia total do quadril (ATQ) é um dos procedimentos ortopédicos mais comuns e bem sucedidos realizados anualmente. ${ }^{1}$ Embora o sangramento na maioria dos casos seja facilmente controlado, anormalidades vasculares como malformações arteriovenosas (MAVs) e fístulas arteriovenosas (FAVs) podem levar a dificuldade em alcançar hemostasia. ${ }^{2}$ Embora raras, os cirurgiões devem estar cientes dessas malformações vasculares, pois o reconhecimento e a intervenção precoces são fundamentais na prevenção de isquemias com risco de membros ou hemorragia com risco de vida. ${ }^{3,4}$ A partir do conhecimento atual, este é o primeiro caso relatado de ATQ primária em uma mulher idosa utilizando uma abordagem anterior direta (AAD) com a descoberta intraoperatória de uma MAV congênita e posterior desenvolvimento de uma FAV pós-operatória com hematomas sintomáticos associados. $\mathrm{O}$ caso foi gerenciado com sucesso por embolia seletiva de bobinas endovasculares e transfusões de sangue.

\section{Relato de caso}

Uma mulher de 81 anos foi apresentada ao pronto-socorro com fortes dores no quadril esquerdo após uma queda. As radiografias mostraram fratura no pescoço do fêmur, e a paciente optou por se submeter a ATQ (-Fig. 1). Note-se que não houve evidência de anormalidades vasculares ou comprometimento na extremidade inferior esquerda. Pulsos estavam presentes e iguais por toda parte e palor estava normal.
Uma abordagem clássica anterior ao quadril foi feita. Após a elevação do músculo reto, foram identificados e coagulados os ramos circunflexos ascendentes. Vasos sanguíneos aparentemente anormais também estavam presentes nesta área. Estes vasos proporcionaram dificuldade em alcançar hemostasia, resultando inicialmente em $\sim 300$ a $400 \mathrm{~mL}$ de perda de sangue durante este processo. A hemostasia acabou sendo alcançada, e o restante da ATQ foi realizado sem

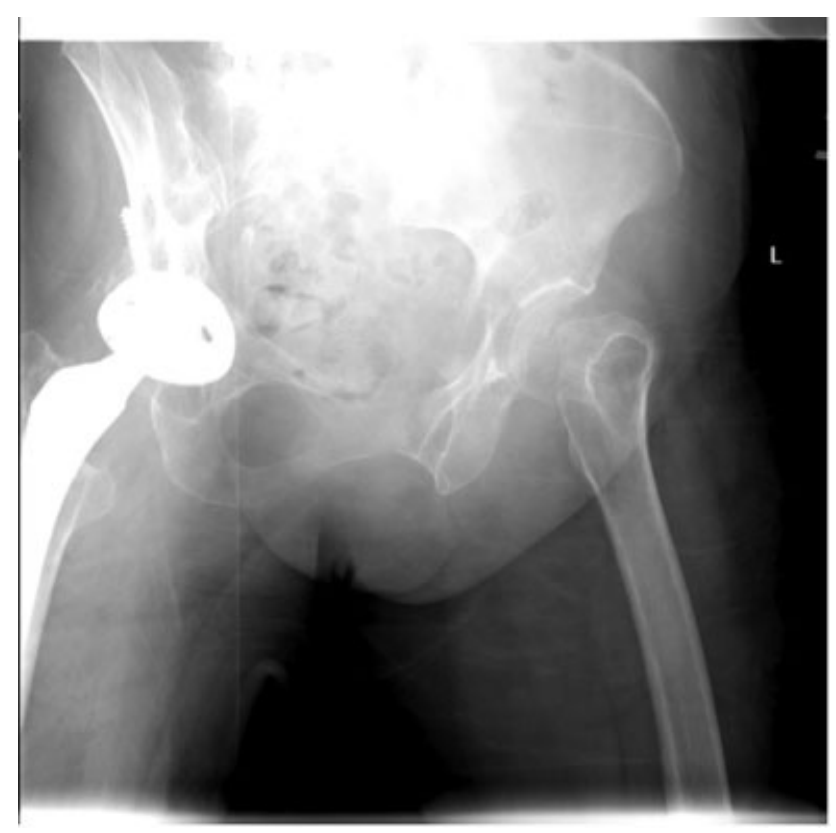

Fig. 1 Radiografia do quadril esquerdo na apresentação mostrando fratura no pescoço do fêmur. 


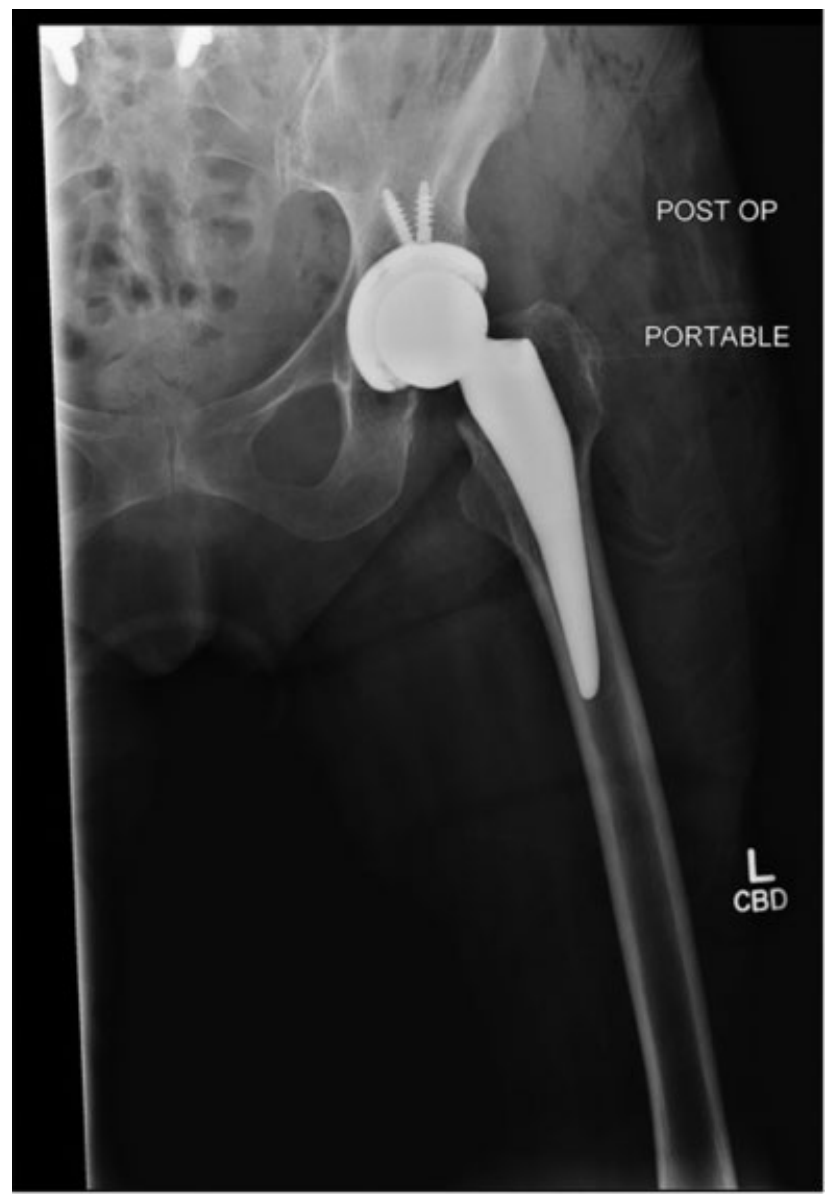

Fig. 2 Radiografia pós-operatória mostrando colocação bem sucedida da artroplastia total do quadril esquerdo.

intercorrências (-Fig. 2). Durante o procedimento, a paciente recebeu uma unidade de sangue. Ela estava estável imediatamente após a operação.

Hemoglobina e hematócrito $(\mathrm{H} / \mathrm{H})$ pós-operatórios foram 8,8 e 26 , abaixo dos 12,0 e 36 medidos no pré-operatório. A paciente recebeu uma unidade de sangue tanto no $2^{\circ}$ quanto no $3^{\circ}$ dias pós-operatórios, pois o $\mathrm{H} / \mathrm{H}$ continuou a cair para $6,5 / 19$. A paciente então começou a reclamar do aumento do inchaço da parte baixa da perna esquerda para a parte proximal da coxa. A tomografia computadorizada (TC) revelou um hematoma de $10 \mathrm{~cm}$ dentro da musculatura adutora esquerda e outro hematoma de 7,3 cm dentro do músculo iliopsoas esquerdo. 0 ultrassom (US) da extremidade inferior esquerda não identificou um trombo bruto, limitado devido à grande quantidade de inchaço. A angiografia da tomografia revelou extravasão ativa com hematoma de coxa esquerda associado predominantemente localizado no compartimento adutor.

Após esses resultados, a radiologia intervencionista (RI) determinou que a extravasão ativa decorreu de um ramo da artéria femoral profunda esquerda. Também foi identificada uma FAV nessa área (-Fig. 3). O sangramento foi resolvido com embolização de bobina seletiva bem sucedida (-Fig. 4). A $\mathrm{H} / \mathrm{H}$ diminuiu por mais 2 dias, exigindo 3 transfusões

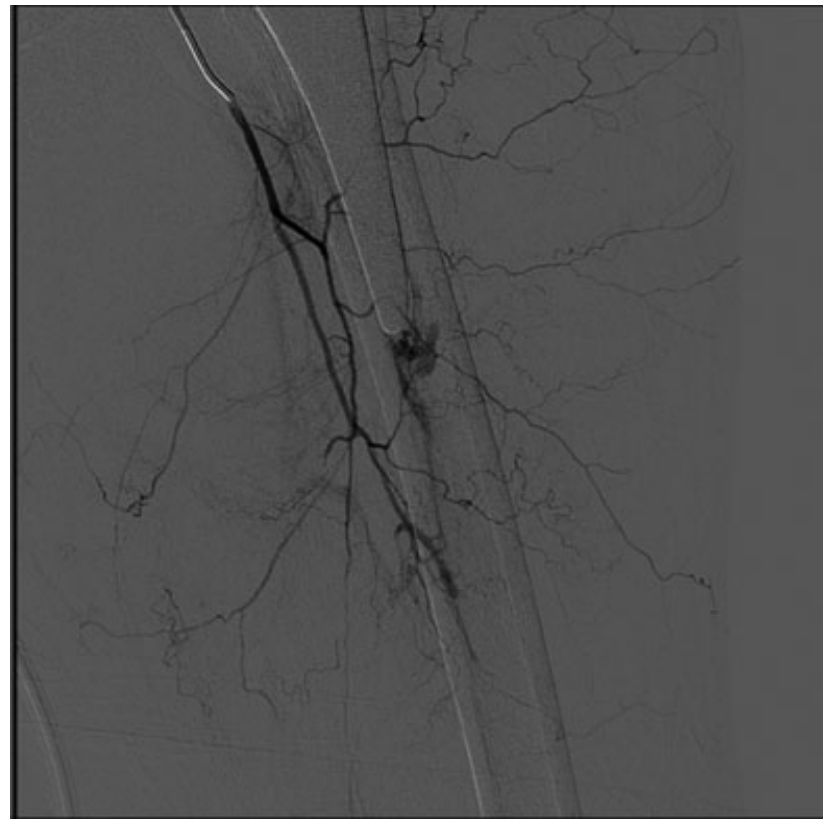

Fig. 3 Angiografia mostrando extravasação ativa e fístula arteriovenosa da artéria femoral profunda esquerda.

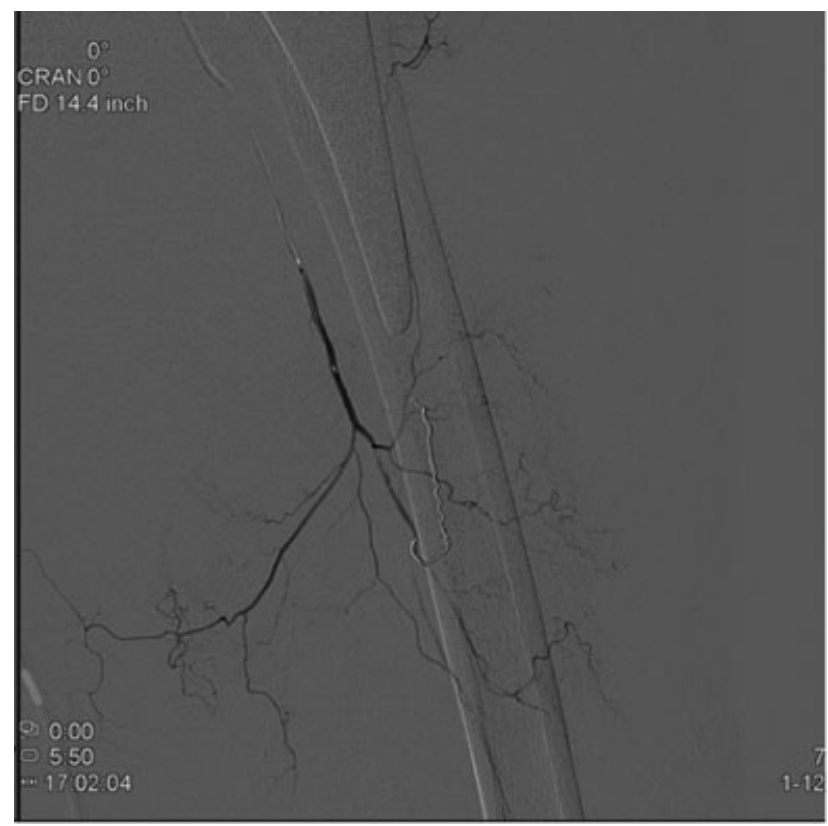

Fig. 4 Embolização pós-bobina mostrando resolução de extravasação e fístula.

adicionais. Na sequência, o $\mathrm{H} / \mathrm{H}$ aumentou constantemente até que ela foi capaz de manter hemostasia.

No quarto dia após a embolização da bobina, a paciente relatou redução da dor e inchaço e continuou a melhorar atéa alta no $8^{\circ}$ dia pós-operatório.

\section{Discussão}

A ATQ é amplamente aceita como uma opção de tratamento segura e eficaz para melhorar a dor e a funcionalidade em muitos pacientes. ${ }^{1,2}$ Ocorrendo em $<1 \%$ dos procedimentos 
de ATQ a infrequência das lesões arteriais torna desafiador que os cirurgiões reconheçam e diagnostiquem adequadamente durante o curso perioperatório. ${ }^{2,5,6}$

As lesões vasculares associadas à ATQ ocorrem devido à proximidade do campo de operação com os principais vasos sanguíneos da pelve e da extremidade inferior, ${ }^{3,4}$ enquanto são expostos a força excessiva por retráteis, preparações acetabulares de remato e marteladas. ${ }^{6}$ Isso é especialmente amplificado em uma população com maiores taxas de aterosclerose e outras doenças vasculares periféricas. ${ }^{7}$

A MAV é uma anomalia vascular descrita como uma rede congênita de comunicação direta entre vasos arteriais e venosos, enquanto a FAV refere-se a uma lesão semelhante, mas adquirida, quase exclusivamente associada a traumas penetrantes como a cirurgia., ${ }^{3,8}$ Tais vasos carregam altas taxas de fluxo vascular que podem resultar em hemorragia excessiva se rompidos. ${ }^{8}$ Essas conexões anormais podem facilmente romper na fase aguda, apresentando hemorragia e formação de hematoma no pós-operatório. ${ }^{3,8}$

No presente caso, a descoberta de vasos sanguíneos incomuns na perna anteromedial combinada com a perda excessiva de sangue e a dificuldade em alcançar a hemostasia foram todos diagnósticos de uma MAV subjacente. Casos semelhantes de MAV maligna e FAV ao redor do quadril foram descritos, mas todos foram descobertos anos após a cirurgia primária do quadril. ${ }^{3,8,9}$ Este é o primeiro caso relatado de uma MAV congênita não diagnosticada anteriormente encontrada durante a ATQ primária com desenvolvimento associado de FAV sintomática no pós-operatório imediato.

Embora a prevalência de malformações vasculares ao redor da articulação do quadril seja pouco documentada na literatura, tem sido relatado que mais da metade das MAVs de tecido mole afetam os vasos femorais ou ilílicos. ${ }^{9}$ Curiosamente, o presente caso e os demais casos referenciados na revisão bibliográfica envolveram lesões da artéria femoral profunda. ${ }^{3,8-10}$ Embora sejam necessários estudos mais baseados em evidências para quantificar a verdadeira predisposição de complicações vasculares em vasos específicos, entender quais vasos estão em maior risco de má formação pode auxiliar os cirurgiões no estabelecimento de uma abordagem cirúrgica superior com risco reduzido de complicações hemorrágicas.

Independentemente dos vasos envolvidos, é importante que os médicos reconheçam a apresentação clínica do sangramento no sítio perioperatório. Os sintomas do sangramento ativo incluem dor localizada, inchaço pulsante e desenvolvimento de instabilidade hemodinâmica. ${ }^{2,4}$ Embora a hemostasia possa ser alcançada intraoperatoriamente, recomenda-se a consulta de RI pós-operatória para todos os encontros suspeitos de MAV para garantir a resolução completa da lesão.

Embora a intervenção cirúrgica aberta possa permitir visualização direta, ela pode resultar em danos adicionais à lesão vascular. ${ }^{7}$ Mais recentemente, tem havido uma tendência crescente de gestão endovascular de casos semelhantes por meio da embolização transcateter. ${ }^{4,7}$ Esses procedimentos minimamente invasivos são altamente efica- zes para o tratamento de complicações arteriais de ATQ e têm mostrado produzir desfechos equivalentes sem expor os pacientes aos riscos adicionais da cirurgia. ${ }^{2,5}$

Embora raras, anomalias vasculares como MAV ou FAV podem causar sérias complicações durante o curso perioperatório da ATQ. Este é o primeiro caso relatado de ATQ primária usando uma AAD com a descoberta intraoperatória de uma MAV congênita e posterior desenvolvimento de uma FAV com hematomas associados no pós-operatório imediato, gerenciado com sucesso endovascularmente por embolização de bobina seletiva. A conscientização dessas malformações vasculares e das estratégias atuais de tratamento é fundamental na prevenção de hemorragias graves.

\section{Suporte Financeiro}

Não houve suporte financeiro de fontes públicas, comerciais, ou sem fins lucrativos.

\section{Conflito de Interesses}

Os autores declaram que não têm conflito de interesses.

\section{Referências}

1 Talia AJ, Coetzee C, Tirosh O, Tran P. Comparison of outcome measures and complication rates following three different approaches for primary total hip arthroplasty: a pragmatic randomised controlled trial. Trials 2018;19(01):13

2 Erschbamer M, Den Hollander J, Sauter D, Erhardt J, Hechelhammer L, Külling F. Endovascular embolisation is a successful and safe treatment for post-operative arterial complications after total hip arthroplasty and revision surgery. Int Orthop 2016;40 (08):1577-1582

3 Chun HJ, Osuga K, Sugihara E, Tsukaguchi I, Ohsawa S, Tsuda K. Transarterial embolization of large complex arteriovenous fistula associated with hip joint surgery. Jpn J Radiol 2010;28(06): 489-492

4 Cheng H, Shin JH, Yoon HK, et al. Retrospective 12-year study of the safety and efficacy of transcatheter arterial embolization for managing bleeding complications following hip surgery. Cardiovasc Intervent Radiol 2014;37(06):1464-1468

5 Troutman DA, Dougherty MJ, Spivack AI, Calligaro KD. Updated strategies to treat acute arterial complications associated with total knee and hip arthroplasty. J Vasc Surg 2013;58(04): 1037-1042

6 Calligaro KD, Dougherty MJ, Ryan S, Booth RE. Acute arterial complications associated with total hip and knee arthroplasty. J Vasc Surg 2003;38(06):1170-1177

7 Kickuth R, Anderson S, Kocovic L, Ludwig K, Siebenrock K, Triller J. Endovascular treatment of arterial injury as an uncommon complication after orthopedic surgery. J Vasc Interv Radiol 2006;17 (05):791-799

8 Lee HJ, Kwon BS, Kwon JH, et al. Arteriovenous malformation incidentally found by ultrasonography in a thigh hematoma after contusion. Ann Rehabil Med 2012;36(02):287-290

9 Winemaker MJ, Boucher MA, de V deBeer J, Colterjohn N, Petruccelli D. Arteriovenous malformation mimicking femoral osteolysis after total hip arthroplasty. J Arthroplasty 2001;16(03): 394-399

10 Lee YO, Hong SW. Treatment of large arteriovenous malformation in right lower limb. Korean J Thorac Cardiovasc Surg 2014;47(01): 66-70 